\title{
Extended Bounds of Beatty Sequence Associated with Primes
}

\author{
F. Deraman, S. H. Sapar, M. A. M. Johari, K. A. Mohd Atan, A. F. N. Rasedee
}

\begin{abstract}
This paper discusses on the estimation of character sums with respect to non-homogeneous Beatty sequences, $\lfloor\alpha p+\beta\rfloor$ over prime $p \leq m$ where $\beta \in \mathbb{R}, x \in \mathbb{N}$ and $\alpha>0$ is irrational. In particular, the bounds is found by extending several properties of character sums associated with composite moduli over prime. As a result, the bound of $\varphi(m)^{\frac{1}{4}} \# \mathcal{P}^{\frac{1}{2}}+\# \mathcal{P} D_{\alpha, \beta}(P)$ is deduced.
\end{abstract}

Index Terms: Beatty sequences, character sum, composite moduli, discrepancy.

\section{INTRODUCTION}

Beatty sequences was introduced by [1] in the form of $\lfloor\alpha n\rfloor$ where $\alpha$ is an irrational and $n$ is natural number.

There are two types of sequences which are homogeneous and non-homogeneous Beatty sequences. In [2] proved that if $\alpha, \beta$ are positive irrational numbers with $\alpha>1$, then the disjoint union of $\boldsymbol{B}(\alpha)$ and $\boldsymbol{B}(\beta)$ are $\mathbb{N}$ if and only if

$$
\frac{1}{\alpha}+\frac{1}{\beta}=1
$$

for $S(\alpha)=\{[n \alpha]\}$ where $n \in \mathbb{N}$. This sequences come along with the compliment sequences which stated in The Theory of Sound [3]. The sequences consisting of the positive integers that are not in the sequence, is itself a Beatty sequence generated by a different irrational number. [4] give proved precisely three conditions (the two sequences of the theorem are disjoint, no integer is absent from both sequences and each sequence consists of distinct elements) of the classical result due to Beatty.

Non-homogeneous Beatty sequence is the sequence of integers $[\alpha n+\beta]$ where $\alpha, \beta$ be a fixed reals numbers in the set

$$
\boldsymbol{B}_{\alpha, \beta}=\{\lfloor\alpha n+\beta\rfloor: n=1,2,3, \ldots\} .
$$

The non-homogeneous Beatty sequences however have been treated by many authors such as [5], [6] and [7]. The study of the distribution of prime numbers in Beatty sequences is started in 1970s. Then, in 2000s some works continued hence there are new approach to improve the estimation of remainder terms in asymptotic formulas. A wide range of study concern on the sums of the values of

Revised Manuscript Received on September 22, 2019.

F. Deraman, Institut Kejuruteraan Matematik, Universiti Malaysia Perlis.

S. H. Sapar, Institut Penyelidikan Matematik, Universiti Putra Malaysia. M. A. M. Johari, Jabatan Matematik, Fakulti Sains, Universiti Putra Malaysia.

K. A. Mohd Atan, Institut Penyelidikan Matematik, Universiti Putra Malaysia.

A. F. N. Rasedee, Fakulti Ekonomi dan Muamalat, Universiti Sains Islam Malaysia. arithmetical functions over Beatty sequences.

In this study we will apply the approach introduced by [8], namely Vinogradov's method. This method give estimation of sequences by using fundamental principle is that possible to estimate effectively sums of the form

$$
\sum_{u} \sum_{v} e(\alpha u v)
$$

under vary varied conditions of summation on $u$ and $v$. The basic properties of Cauchy inequalities and Fourier series from this method are applied in [9] to estimate bounds of double character sums.

The complement of this result is obtained in [10] which gives for a prime $p$ the least positive quadratic non-residue modulo $p$ among the terms of Beatty sequence is of size at most $p^{1 /\left(4 e^{1 / 2}\right)+o(1)}$. However, the method used in [10] is very different and cannot be used to find bound the sum $S_{k}(\alpha, \beta, \chi ; N)$ of this study.

In [11] and [12], they used the following character sums

$$
S_{p}(\alpha, \beta, \chi ; N)=\sum_{n \leq N} \chi(\lfloor\alpha n+\beta\rfloor)
$$

where $\alpha$ is irrational and $\chi$ is a non principal character sum modulo $p$. The sum $S_{p}(\alpha, \beta, \chi ; N)$ is studied for special case of primes and estimated nontrivial for $N \geq p^{1 / 3+\epsilon}$, where $\epsilon>0$, respectively. They obtain the results on uniform distribution of primitive roots which improved the result in [13]. The estimation of bounds is stronger on the size of the least quadratic non residue by using character sums approached.

In this paper, we investigate for the cases of composite moduli $m$. Then, the results on the estimation of the bound of sums $S_{m}(\alpha, \beta, \chi ; P)$ are in primes numbers which is highlighted in an open problem listed by [14]. That is we consider non-homogeneous Beatty sequences over primes. It is constructed in the form of

$$
\boldsymbol{B}_{\alpha, \beta}=\{\lfloor\alpha p+\beta\rfloor: p=2,3,5, \ldots\} .
$$

Firstly, consider the sum of the form

$$
S_{m}(\alpha, \beta, \chi ; P)=\sum_{p \leq P} \chi(\lfloor\alpha p+\beta\rfloor),
$$

where $\alpha$ is an irrational, $p$ is a primes and $\chi$ is a non trivial multiplicative character modulo a composite number. We applied a method given by [11] and [9] in order to estimate the bound of distributions of non-homogeneous Beatty sequences. The result yield explicit bounds on error term. By using this method and identify the cardinality of the primes sets, we will established a new results [15]. 


\section{A. Notation}

The notations such as $U=O(V), U \ll V$, and $V \gg U$ is applied equivalent to the assertion $|U| \leq c V$ for some constant $c>0$. The constants symbols $O, \gg$ and $\ll$ may depend on the real number $\alpha$ but are absolute otherwise. A function which tends to zero and depends only on $\alpha$ is denoted as $o(1)$. It is important to note that our bounds are uniform with respect to all of the other parameters, in particular with respect to $\beta$.

The letters $k, m$, and $n$ with or without subscripts always denote non-negative integers.

If $x$ is a real number the symbols $\lfloor x\rfloor,\lceil x\rceil$ denote the greatest integer $\leq x$ and the least integer $\geq x$, respectively. The fractional part $\lfloor x\rfloor-x$ of $x$ is denoted by $\{x\}$. The notation of distance from the real number $x$ is denoted as $\|x\|=\min _{n \in \mathbb{Z}}|x-n|$.

\section{Preliminaries}

Our result is applied to some familiar notation from the theory of Diophantine approximations which describes the class of real numbers $\alpha$. For an irrational number $\alpha$, we define its type $\tau$ by the relation [12]

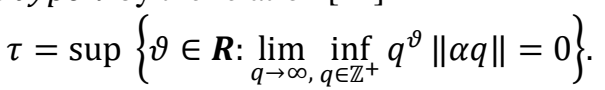

By using Dirichlet's approximation theorem, it is easy to see that $\tau \geq 1$ for every irrational $\alpha$. [16] and [17] assert that $\tau=1$ for almost all real (in the sense of the Lebesgue measure) and all irrational algebraic numbers $\alpha$, respectively. We remark that the number $\mu=\tau+1$ is called the irrationality measure of $\alpha$, or the LiouvilleRoth constant.

The discrepancy, $D$ of a sequence is an introduced quantity that measures the deviation of the sequence from an ideal distribution. The discrepancy, $D$ of a sequence of $M$ (not necessarily distinct) real numbers $\gamma_{1}, \gamma_{2}, \ldots, \gamma_{M} \in[0,1)$ is defined by the relation

$$
D=\sup _{I \subseteq[0,1)}\left|\frac{V(I, M)}{M}-\right| I||
$$

where the supremum is taken over all subintervals $I=(a, b)$ of the interval $[0,1), V(I, M)$ is the cardinality of the set $\left\{1 \leq m \leq M: \gamma_{m} \in I\right\}$ and $|I|$ is the length of $I$. [11].

We also need the following elementary statement from

Lemma 1. Let $\alpha$ be a fixed irrational number. Then, for every positive integer $M$ and real number $\delta \in(0,1]$, there exists a real number $\gamma$ such that

$$
\#\{m \leq M:\{\alpha m+\gamma\}<\delta\} \geq 0.5 M \delta \text {. }
$$

Proof: Suppose $e(x)=\exp (2 \pi i x)$ for all $x \in \mathbb{R}$. Note that $e(x)=e(y)$ if and only if $\{x\}=\{y\}$. Since $\alpha$ is irrational, the numbers $e(\alpha m)$ have distinct arguments for $m=1,2, \ldots, M$.

Let $L=\left\lfloor\delta^{-1}\right\rfloor$, and

$$
S_{j}=\left\{m \leq M: \frac{2 \pi j}{L} \leq(e(\alpha m))<\frac{2 \pi(j+1)}{L}\right\}
$$

where $0 \leq j \leq L-1$.

Since $\{1,2, \ldots, M\}$ is the disjoint union of the sets $S_{j}, j=$ $0,1,2, \ldots, L-1$. By the pigeonhole principle there exists an index $j$ for which

$$
\# S_{j} \geq \frac{M}{L}=\frac{M}{\rfloor \delta^{-1}\lfloor} \geq 0.5 M \delta .
$$

Let $\gamma=-\frac{j}{L}$ and for each $m \in S_{j}$, we have

$$
\begin{gathered}
\{\alpha m+\gamma\}=\frac{1}{2 \pi} \arg (e(\alpha m+\gamma)) \\
=\frac{1}{2 \pi} \arg \left(e\left(\alpha m-\frac{2 \pi j}{L}\right)\right)<\frac{1}{L} \leq \delta,
\end{gathered}
$$

which finishes the proof.

\section{RESUlTS AND Discussion}

In this section, we give a result of an open problem (Number 35) in [14] on distribution of composite moduli over prime for non-homogeneous Beatty sequences. The following subsection discuss the properties of character sums extended to composite moduli and the bounds of distributions of the sequences associated to primes.

\section{A. On Some Properties of Character Sum}

The following lemmas will discuss on the properties of character sums extended to composite moduli. Consider $m$ a composite number with primitive elements.

Let $\mathbb{F}_{m}$ be a finite group of order $\varphi(m)$ and $g$ be a cyclic subgroup of $\mathbb{F}_{m}$ with order $\mu$.

Lemma 2:A multiplicative character of $\mathbb{F}_{m}^{*}$ is,

$$
\chi_{j}\left(g^{k}\right)=e^{\frac{2 \pi i j k}{\mu}} \text { where } k=0,1, \ldots, \varphi(m)-1
$$

where $g$ is a fixed primitive element of $\mathbb{F}_{m}$ with order $\mu$ and $j$ is a fixed integer, $0 \leq j \leq \varphi(m)-1$.

Proof. By the hypothesis, $\chi(g)$ must be a $\mu$-th roots of unity. Therefore, $\chi_{j}(g)=e^{\frac{2 \pi i j}{\mu}}$ for some $j, 0 \leq j \leq n-1$. It follows that $\chi(g)=\chi_{j}$. There exists, $\mathbb{F}_{m}$ that consists exactly the characters $i_{0}, \chi_{1}, \ldots, \chi_{\varphi(m)-1}$.

By using the ortogonality relations that applied to additive or multiplicative characters sums to $\mathbb{F}_{m}$ will yield several fundamental identities.

Lemma 3. For additive character $\chi_{a}$ and $\chi_{b}$,

$$
\sum_{c \in \mathbb{F}_{m}} \chi_{a}(c) \overline{\chi_{b}(c)}= \begin{cases}\varphi(m)+1 & \text { if } a=b \\ 0 & \text { if } a \neq b\end{cases}
$$

For multiplicative character, if , $d \in \mathbb{F}_{m}^{*}$, then

$$
\sum_{\chi} \chi_{c}(a) \overline{\chi_{c}(b)}=\left\{\begin{array}{cc}
\varphi(m) & \text { if } a=b \\
0 & \text { if } a \neq b .
\end{array}\right.
$$

where the sum is extended over all multiplicative character $\chi$ of $\mathbb{F}_{m}$.

Proof: Let $q_{i}$ be primitive roots from $\mathbb{F}_{m}$ of order $\varphi(m)$ where $0 \leq i \leq \varphi(m)-1$.

Then, we have cyclic subgroup $\left\{q_{1}, q_{2}, \ldots, q_{\varphi(m)-1}\right\}$ of order $\left\{\mu_{1}, \mu_{2}, \ldots, \mu_{\varphi(m)-1}\right\}$, respectively. After that, we choose only primitive roots of $q_{i}$ that satisfy $\operatorname{gcd}\left(m, q_{i}\right)=1$.

By using the function from Lemma 2, 


$$
\chi_{j}\left(q_{i}^{k}\right)=e^{\frac{2 \pi i j k}{\mu}} \quad \text { where } \quad k=0,1, \ldots, \varphi(m)-1
$$

will give us all additive and multiplivative character of $\mathbb{F}_{m}$ for any composite moduli.

Lemma 4. Let $a, b$ from $\mathbb{F}_{m}$ and $c$ from $\mathbb{F}_{m}^{*}$. If $c+a=$ $d_{1}$ and $c+b=d_{2}$. Then the nontrivial multiplicative character of $\mathbb{F}_{m}$ is given by,

$$
\sum_{\chi} \chi\left(d_{1}\right) \bar{\chi}\left(d_{2}\right)= \begin{cases}0 & \text { if } d_{1} \neq d_{2} \\ \varphi(m)+1 & \text { if } d_{1}=d_{2}\end{cases}
$$

Proof: Given that $c+a=d_{1}$ and $c+b=d_{2}$. There exist additive properties of identity element as trivial case which $\chi(d)$ is different from 0 for every $a$ in $G$.

Suppose for some $d \in \mathbb{F}_{m}$ is $\chi(d)=0$. Then $\chi(e)=$ $\chi\left(d d^{-1}\right)=\chi(d) \chi\left(d^{-1}\right)=0$. From Lemma 2 it is obviously shown $\chi(e) \neq 0$. Thus for this case we consider $\chi(e)=1$.

Consider $\operatorname{gcd}\left(d_{1_{r}}, \mathrm{~m}\right)=1$ and $\operatorname{gcd}\left(d_{2_{r}}, \mathrm{~m}\right)=1$. Then we will have $d_{1_{r}}$ and $d_{2_{r}}$ be primitive roots from $\mathbb{F}_{m}$ of order $\varphi(m)$ where $0 \leq r \leq \varphi(m)-1$.

Then only consider primitive elements of $\operatorname{gcd}\left(d_{1_{r}}, \mathrm{~m}\right)=1$ and $\operatorname{gcd}\left(d_{2_{r}}, \mathrm{~m}\right)=1$ from $\mathbb{F}_{m}^{*}$. By Lemma 2 , then the lemma holds.

The case of $m$ is an odd prime was solved by [11].

\section{B. Bounds of Beatty Sequence Associated with Prime}

Theorem I discuss on the bound of distribution of constructed by applying discrepancy, $D_{\alpha, \beta}(P)$ of the sequence of fractional parts in the form of

$$
\{\{\alpha p+\beta\}: p=2,3, \ldots, P\}
$$

Theorem I: Let $\alpha$ be a fixed irrational number, $\beta$ be any real number and $m$ is any composite number with primitive elements. Then, for positive primes $P \leq m$, set of prime $\mathcal{P}$ and non-trivial multiplicative characters $\chi(\bmod \mathrm{m})$, the following bound holds:

$$
S_{m}(\alpha, \beta, \chi ; P) \ll \varphi(m)^{\frac{1}{4}} \# \mathcal{P}^{\frac{1}{2}}+\# \mathcal{P} D_{\alpha, \beta}(P) .
$$

Proof: The proof of this theorem divided into two parts. The first part deals with discrepancy of sequences and character sums and the second part will gives more details on character sums in composite moduli associated with primes.

The prove of the first part is as follows:

Let $k \leq P \in$ primes, $\triangle \in(0,1] \in \mathbb{R}$ and $\gamma \in \mathbb{R}$.

$\mathcal{P}_{\gamma}=\{2 \leq p \leq P:\{\alpha p+\beta-\gamma\}<1-\triangle\}$

$\mathcal{K}_{\gamma}=\{2 \leq k \leq K:\{\alpha k+\gamma\}<\triangle\}$

Suppose

$$
\mathcal{P}_{\gamma}^{c}=\{2,3,5 \ldots, P\} \backslash \mathcal{P}_{\gamma}
$$

From (1), we have $V(I, P)=\# \mathcal{P}_{\gamma}^{c}$ is the cardinality of the set $\left\{2 \leq p \leq P: \gamma_{p} \in I\right\}$ and $|I|=|\triangle|$ is the length of $I$.

Then we have,

$$
D_{\alpha, \beta}(P)=\sup _{I \subseteq[0,1)}\left|\frac{\# \mathcal{P}_{\gamma}^{c}}{\# \mathcal{P}}-\right| \triangle|| .
$$

Thus,

$$
\# \mathcal{P}_{\gamma}^{c}=O\left(\# \mathcal{P} D_{\alpha, \beta}(P)\right)+\# \mathcal{P}|\triangle|
$$
quadaratic residue by using character sums. The result is

For some choice of $\gamma \in \mathbb{R}$, from Lemma 1

$$
\# \mathcal{K}_{\gamma} \geq 0.5 K \triangle \text {. }
$$

By fixing $\gamma$, let $\mathcal{P}_{\gamma}=\mathcal{P}, \mathcal{P}_{\gamma}^{c}=\mathcal{P}^{c}$ and $\mathcal{K}_{\gamma}=\mathcal{K}$.

We have for every $k \in \mathcal{K}$,

$$
\begin{gathered}
S_{m}(\alpha, \beta, \chi ; P)=\sum_{p \leq P} \chi(\lfloor\alpha(p+k)+\beta\rfloor)+O(k) \\
=\sum_{p \leq P} \chi(\lfloor\alpha(p+k)+\beta\rfloor)+O(K) \\
=\sum_{p \in \mathcal{P}} \chi(\lfloor\alpha(p+k)+\beta\rfloor)+O\left(K+\# \mathcal{P}^{c}\right) .
\end{gathered}
$$

It can be shown that,

$$
S_{m}(\alpha, \beta, \chi ; P)=\frac{W}{\# \mathcal{K}}+O\left(K+\# \mathcal{P}^{c}\right),
$$

where

$$
W=\sum_{p \in \mathcal{P}} \sum_{k \in \mathcal{K}} \chi(\lfloor\alpha(p+k)+\beta\rfloor) .
$$

For any $p \in \mathcal{P}$ and some $k \in \mathcal{K}$, we have

$$
\begin{aligned}
& \lfloor\alpha(p+k)+\beta\rfloor=\alpha(p+k)+\beta-\{\alpha(p+k)+\beta\} \\
& =(\alpha p+\beta-\gamma)+(\alpha k+\gamma)-\{\alpha p+\beta-\gamma\} \\
& -\{\alpha k+\gamma\} \\
& =\lfloor\alpha p+\beta-\gamma\rfloor+\lfloor\alpha k+\gamma\rfloor .
\end{aligned}
$$

Since $P \leq m$, we have

$$
\#\{p \in \mathcal{P}:\lfloor\alpha p+\beta-\gamma\rfloor \equiv s(\bmod m)\}=O(1)
$$

uniformly for all $s \in \boldsymbol{Z}$.

Therefore, by applying the Cauchy inequality we obtain $|W|^{2}$

$$
\begin{aligned}
& \ll \# \mathcal{P} \sum_{p \in \mathcal{P}}\left|\sum_{k \in \mathcal{K}} \chi(\lfloor\alpha p+\beta-\gamma\rfloor+\lfloor\alpha k+\gamma\rfloor)\right|^{2} \\
\ll & \# \mathcal{P} \sum_{s=1}^{m}\left|\sum_{k \in \mathcal{K}} \chi(s+\lfloor\alpha k+\gamma\rfloor)\right|^{2} \\
& =\# \mathcal{P} \sum_{k, l \in \mathcal{K}} \sum_{s=1}^{m} \chi(s+\lfloor\alpha k+\gamma\rfloor) \bar{\chi}(s+\lfloor\alpha l+\gamma\rfloor) .
\end{aligned}
$$

Now we will prove the second part. From Lemma 4, the inner sum takes only two possible values. That is

$$
\begin{gathered}
\left.\sum_{s=1}^{m} \chi(s+\lfloor\alpha k+\gamma\rfloor)\right) \bar{\chi}(s+\lfloor\alpha+\gamma\rfloor) \\
=\left\{\begin{array}{cr}
\varphi(m) & \text { if }\lfloor\alpha k+\gamma\rfloor \equiv\lfloor\alpha l+\gamma\rfloor(\bmod m) \\
0 & \text { if otherwise. }
\end{array}\right.
\end{gathered}
$$

Since $K \leq m$, the congruence $\lfloor\alpha k+\gamma\rfloor \equiv\lfloor\alpha l+$ $\gamma\rfloor(\bmod m)$ occurs for at most $O(\# \mathcal{K})$ pairs $k, l \in \mathcal{K}$. Therefore, it follows that

$|W|^{2} \ll \# \mathcal{P}\left((\# \mathcal{K})^{2}+\varphi(m) \cdot \# \mathcal{K}\right) \ll \varphi(m) \# \mathcal{P} \cdot \# \mathcal{K}$.

Subtituting this bound in (4) and by using (2) and (3), we obtain

$$
\begin{aligned}
& S_{m}(\alpha, \beta, \chi ; P) \ll \sqrt{\frac{\varphi(m) \# \mathcal{P}}{K \triangle}}+ \\
& K+\# \mathcal{P} \triangle+\# \mathcal{P} D_{\alpha, \beta}(P) \\
& \quad \text { where }
\end{aligned}
$$




$$
K=\left\lfloor\varphi(m)^{\frac{1}{4}} \# \mathcal{P}^{\frac{1}{2}}\right\rfloor \text { and } \quad \triangle=\frac{\varphi(m)^{\frac{1}{4}}}{\# \mathcal{P}^{\frac{1}{2}}}
$$

Thus, the theorem holds.

As an illustration, if we have $p=23, \alpha=\sqrt{3}, \beta=1.1$, and $\gamma=0.3$. Then $\alpha p+\beta-\gamma=19.3526$. The fractional of $\{\alpha k+\gamma\}<0.3628$.

Suppose $k=11$, then we will have $\lfloor\alpha(p+k)+\beta\rfloor=$ $\lfloor 59.6897\rfloor=59$ and $\lfloor\alpha p+\beta-\gamma\rfloor+\lfloor\alpha k+\gamma\rfloor=$ $[40.6372\rfloor+\lfloor 19.3526\rfloor=59$.

Thus it is obvious satisfied the representation of $\lfloor\alpha(p+$ $k)+\beta\rfloor=\lfloor\alpha p+\beta-\gamma\rfloor+\lfloor\alpha k+\gamma\rfloor$.

\section{CONCLUSION}

From this research, we found that the bound of non homogeneous Beatty sequences on distribution of composite moduli over primes case is less than $\varphi(m)^{\frac{1}{4}} \# \mathcal{P}^{\frac{1}{2}}+$ $\# \mathcal{P} D_{\alpha, \beta}(P)$.

\section{ACKNOWLEDGMENT}

This research was supported in part by UPM/700-2/1/GPB/2017/9597900 and Fellowship UniMAP, Malaysia.

\section{REFERENCES}

1. O. Beatty, S. Dunkel, A. Pelletier, F. Irwin, J. L. Riley,P. Fitch, and D. M. Yost, " Problem 3173," American Mathematical Monthly, vol. 33 , no. 3 , p. 159,1926

2. A. Beatty, S. Ostrowski, J. Hypslop, and A. C. Aitken,"Solution to Problem 3173," American Mathematical Monthly, vol. 34, no. 3, pp. 159-160, 1927.

3. J. W. Strutt and B. Rayleigh, "The Theory of Sound," Macmillan, vol. 2nd Edition, New York 1894.

4. A. M. Mercer, "Generalized Beatty Sequences," Intrnat. J. Math. and Math. Si, no. 525, pp. 525-528, 1978.

5. A. M. G. and C. W. Nevans, "Sums of multiplicative functions over a Beatty sequence,” Bull. Austral. Math.Soc., no. 78, pp. 327-334, 2008.

6. J. Cassaigne, E. Duch Ãane, and M. Rigo, "Nonhomogeneous beatty sequences leading to invariant games,"SIAM Journal on Discrete Mathematics, vol. 30, no. 3, p. 1798-1829, 2016.

7. C. Kimberling, "Beatty sequences and trigonometric functions," Integers, vol. 16, no. \#A15, 2016.

8. I. M. Vingradov, " The method of trigonometrical sums in the theory of numbers," Interscience Publisher, London 2004.

9. W. Banks and I. Shparlinski, " Character sums with Beatty sequences on Burgess-type intervals," Analytic number theory: essays in honour of Klaus Roth, Cambridge University Press., pp. 15-21, London 2009.

10. W. D. Banks, M. Z. Garaev, D. R. Heath-Brown, and I. E. Shparlinski, "Density of non-residues in Burgess type intervals and applications," Bulletin of the London Mathematical Society., pp. 88-96, February 2008.

11. W. D. Banks and I. E. Shparlinski, "Non-residuesand primitive roots in beatty sequences," Bull. Austral.Math. Soc., vol. 73, pp. 433-443, 2006.

12. W. D. Banks and I. E. Shparlinski, "Short character sums with beatty sequences," Math. Res. Lett., vol. 13, no. 00, pp. 1-100N, 2006.

13. M. Z. Garaez, "A note on the least quadratic nonresidue of the integer-sequences," Bull. Autral. Math. Soc., vol. 68, pp. 1-11, 2003.

14. I. E. Shparlinski, "Open problems on exponential and character sums," Series on Number Theory and its Applications, World Scientific Publishing, Hackensack, NJ 2010, no. 6, pp. 222-242, 2010.

15. W. D. Banks and I. E. Shparlinski, "Prime divisors inbeatty sequences," Journal of Number Theory, vol. 123,pp. 413-425, 2007.

16. A. Y. Khinchin, "Zur metrischem theorie der diophantischen approximationen," Math. Z., vol. 24, pp. 706-714, 1926.

17. K. F. Roth, "Rational approximations to algebraic numbers," Mathematika, vol. 2, pp. 1-20, 1955.

\section{Authors ProfiLe}

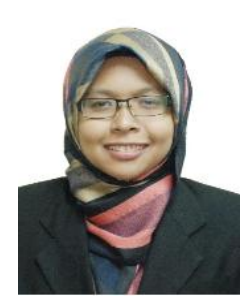

F. Deraman is a fellow at Universiti Malaysia Perlis. In 2009 and 2013 she was honoured Bachelor's degree in Mathematic with Education and Master of Science in Structural Theory of algebras from Universiti Putra Malaysia, respectively. Her research interests are related to exponential sums and classification of Leibniz algebras.

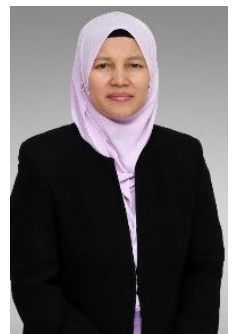

S. H. Sapar is working as an Associate Professor in Department of Mathematics, Faculty of Science, Universiti Putra Malaysia. She holds a Bachelor of Science (Hons.) in Mathematics, Master of Science (Number Theory) and $\mathrm{PhD}$ (Number Theory) form Universiti Putra Malaysia. Her research interests are area related to exponential sums and Diophantine equations. She reviewed a lot of research papers for national and international journals.

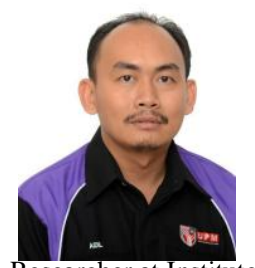

M A M Johari is a lecturer at Department of Mathematics, Faculty of Science, Universiti Putra Malaysia. He obtained his $\mathrm{PhD}$ in Number Theory from Universiti Putra Malaysia on 2013. His research interests are areas related to number of representations and Diophantine equations. He has published more than 10 paper in local and international journal. $\mathrm{He}$ is also a Associate Researcher at Institute for Mathematical Research (INSPEM).

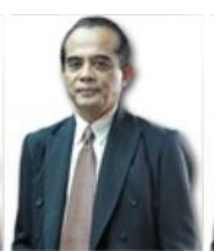

K.A. Mohd Atan is an Emeritus Professor in Universiti Putra Malaysia and President of Malaysian Academy of Mathematical Scientists an adhering organisation to the International Mathematical Union (IMU). He had served the university for more than forty years beginning as an assistant lecturer in the mathematics department and finally as senior Professor and Director of Institute for Mathematical Research of the university. In between he had held the posts of Head of Mathematics Department, Deputy Dean of the Faculty of Science alnd Environmental Studies, Director of Centre of Matriculation Studies and Deputy Vice Chancellor (Academic and Research). $\mathrm{He}$ graduated in pure mathematics with a BA from Universiti Malaya, MA from the University of Western Ontario Canada and $\mathrm{PhD}$ from the Unversity of New South Wales Australia specializing in Number Theory. He has published a total of more than 100 articles in refreed journals and proceedings and made more than 50 paper presentations in conferences as well as authored and co-authored 7 books including translations of English books into the Malay language. He has supervised and co-supervised more than 30 research students leading to the $\mathrm{MSc}$ and $\mathrm{PhD}$ degrees.

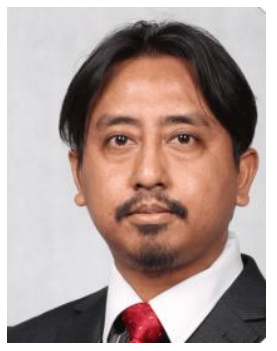

A. F. N. Rasedee completed his $\mathrm{PhD}$ in Pure Mathematics from Universiti Putra Malaysia in 2015 Since then, he has been serving as a senior lecturer at the Faculty of Economics and Muamalat, Universiti Sains Islam Malaysia. He has published over 30 research articles, completed 4 research projects with 9 ongoing and has been appointment as reviewer for numerous indexed journals. He currently holds the post of Big Data Coordinator working under the Chief Information Officer's office. 